PROCEEDINGS OF THE

AMERICAN MATHEMATICAL SOCIETY

Volume 125, Number 12, December 1997, Pages 3533-3539

S 0002-9939(97)04250-0

\title{
FIRST ORDER DIFFERENCE SYSTEM- EXISTENCE AND UNIQUENESS
}

\author{
K. N. MURTY, P. V. S. ANAND, AND V. LAKSHMI PRASANNAM
}

(Communicated by Hal L. Smith)

\begin{abstract}
In this paper, the general solution of the homogeneous matrix difference system is constructed in terms of two fundamental matrix solutions. The general solution of the inhomogeneous matrix difference system is established by the variation of parameters formula. A unique solution of the twopoint boundary value problem associated with the matrix difference system is constructed by applying the QR-algorithm and the Bartels-Stewart algorithm.
\end{abstract}

\section{Introduction}

The theory of difference equations is a lot richer than the corresponding theory of differential equations. For example, a simple difference equation resulting from a first order differential equation may have a phenomenon often called the appearance of "ghost" solutions or the existence of chaotic orbits that can only happen for higher order differential equations. Consequently, the theory of difference equations is interesting in itself and assumes great importance in the present world. The application of the theory of difference equations is already extended to various fields such as numerical analysis, finite element techniques, control theory and computer science. So all these reasons inspired us to consider the matrix difference system

$$
T(n+1)=A(n) T(n) B(n)+F(n)
$$

where $A(n), B(n)$ and $F(n)$ are square matrices of order $s$ whose elements $a_{i j}(n), b_{i j}(n)$ and $f_{i j}(n)$ are real or complex functions defined on $N_{n_{0}}^{+}$and $T(n) \in$ $R^{s \times s}\left(C^{s \times s}\right)$ with components as functions defined on $N_{n_{0}}^{+}$, where

$$
N_{n_{0}}^{+}=\left\{n_{0}, n_{0} \pm 1, \ldots, n_{0} \pm k, \ldots\right\}
$$

with $k \in N^{+}$and $n_{0} \in N, N$ being the set of integers.

The paper is organised as follows. In section 2, we develop the general solution of the non-homogeneous matrix difference system (1.1) and also the general solution of the matrix differential equation

$$
T(n+1)=A(n) T(n) B(n)
$$

in terms of two fundamental matrix solutions of the systems $T(n+1)=A(n) T(n)$

Received by the editors April 17, 1996.

1991 Mathematics Subject Classification. Primary 39A10, 34B05.

(C) 1997 American Mathematical Society 
and $T(n+1)=B^{*}(n) T(n)$. We obtain the general solution of (1.1) by the method of variation of parameters. Section 3 presents criteria for the existence and uniqueness of solutions to two-point boundary value problems associated with (1.1), satisfying the boundary conditions

$$
L_{0} T\left(n_{0}\right)+L_{1} T\left(n_{1}\right)=W
$$

where $n_{0}, n_{1} \in N_{n_{0}}^{+}, n_{0}<n_{1} ; W, L_{0}$ and $L_{1}$ are given constant square matrices of order $s$. Here the Bartels-Stewart algorithm [6] and the QR-algorithm are used as tools to evaluate the constant matrix embedded between two known matrices.

\section{Solution of THE DifFEREnCE SyStem}

In this section, we construct the solution of the homogeneous difference system (1.2) in terms of the fundamental matrix solutions. We establish the general solution of the non-homogeneous difference system (1.1) in terms of fundamental matrix solutions by the method of variation of parameters. We also present the general solution of the initial value problem associated with the matrix difference equation (1.1). We shall denote $\Phi_{1}\left(n, n_{0}\right)$ and $\Phi_{2}\left(n, n_{0}\right)$ as the fundamental matrix solutions of $T(n+1)=A(n) T(n)$ and $T(n+1)=B^{*}(n) T(n)$, respectively. The proof of the following lemma is immediate.

Lemma 2.1. $\Phi_{1}\left(n, n_{0}\right)$ is a fundamental matrix solution of $T(n+1)=A(n) T(n)$ if and only if $\Phi_{1}^{*}\left(n, n_{0}\right)$ is a fundamental matrix solution of $T(n+1)=T(n) A^{*}(n)$.

Theorem 2.1. If $\Phi_{1}\left(n, n_{0}\right)$ and $\Phi_{2}^{*}\left(n, n_{0}\right)$ are fundamental matrix solutions of $T(n+1)=A(n) T(n)$ and $T(n+1)=B^{*}(n) T(n)$, respectively, then any solution $T(n)$ of the homogeneous matrix difference system (1.2) is of the form

$$
\Phi_{1}\left(n, n_{0}\right) C \Phi_{2}^{*}\left(n, n_{0}\right),
$$

where $C$ is an arbitrary constant square matrix of order $s$.

Proof. We seek a solution of (1.2) in the form $T(n)=\Phi_{1}\left(n, n_{0}\right) K(n)$, where $K(n)$ is a square matrix of order $s$ whose elements are functions defined on $N_{n_{0}}^{+}$. Then

$$
\begin{aligned}
\Phi_{1}(n & \left.+1, n_{0}\right) K(n+1)=A(n) \Phi_{1}\left(n, n_{0}\right) K(n) B(n) \\
& \Leftrightarrow \Phi_{1}\left(n+1, n_{0}\right) K(n+1)=\Phi_{1}\left(n+1, n_{0}\right) K(n) B(n) \\
& \Leftrightarrow K(n+1)=K(n) B(n) \\
& \Leftrightarrow K^{*}(n+1)=B^{*}(n) K^{*}(n) .
\end{aligned}
$$

Since $\Phi_{2}^{*}\left(n, n_{0}\right)$ is a fundamental matrix solution of the difference system $T(n+1)=$ $B^{*}(n) T(n)$, it follows that there exists a constant matrix $C_{1}$ of order $s \times s$ such that $K^{*}(n)=\Phi_{2}\left(n, n_{0}\right) C_{1}$ and $T(n)=\Phi_{1}\left(n, n_{0}\right) C \Phi_{2}^{*}\left(n, n_{0}\right)\left(C=C_{1}^{*}\right)$.

Theorem 2.2. Any solution $T(n)$ of the non-homogeneous matrix difference system (1.1) is of the form $\Phi_{1}\left(n, n_{0}\right) C \Phi_{2}^{*}\left(n, n_{0}\right)+\bar{T}(n)$, where $\bar{T}(n)$ is a particular solution of (1.1).

Proof. It can easily be verified that $\Phi_{1}\left(n, n_{0}\right) C \Phi_{2}^{*}\left(n, n_{0}\right)+\bar{T}(n)$ is a solution of (1.1). Now to prove every solution is of the form, let $T(n)$ be any solution of (1.1) and $\bar{T}(n)$ be a particular solution of (1.1). Then it can easily be verified 
that $T(n)-\bar{T}(n)$ is a solution of the homogeneous matrix difference system (1.2). Hence by Theorem 2.1 , we have $T(n)-\bar{T}(n)=\Phi_{1}\left(n, n_{0}\right) C \Phi_{2}^{*}\left(n, n_{0}\right)$. Therefore $T(n)=\Phi_{1}\left(n, n_{0}\right) C \Phi_{2}^{*}\left(n, n_{0}\right)+\bar{T}(n)$.

Theorem 2.3. Any solution of the non-homogeneous matrix difference system (1.1) is of the form $T(n)=\Phi_{1}\left(n, n_{0}\right) C \Phi_{2}^{*}\left(n, n_{0}\right)+\bar{T}(n)$, where $\bar{T}(n)$ is a particular solution of (1.1) and is given by

$$
\sum_{j=n_{0}}^{n-1} \Phi_{1}(n, j+1) F(j) \Phi_{2}^{*}(n, j+1) .
$$

Proof. The general solution of the homogeneous matrix difference system (1.2) is of the form $T(n)=\Phi_{1}\left(n, n_{0}\right) C \Phi_{2}^{*}\left(n, n_{0}\right)$. Let $C$ be a function of $n$ defined on $N_{n_{0}}^{+}$. Since $T(n)$ must satisfy (1.1), we have

$$
\begin{aligned}
\Phi_{1}(n & \left.+1, n_{0}\right) C(n+1) \Phi_{2}^{*}\left(n+1, n_{0}\right)=A(n) \Phi_{1}\left(n, n_{0}\right) C(n) \Phi_{2}^{*}\left(n, n_{0}\right) B(n)+F(n) \\
& \Leftrightarrow \Phi_{1}\left(n+1, n_{0}\right) \Delta C(n) \Phi_{2}^{*}\left(n+1, n_{0}\right)=F(n) \\
& \Leftrightarrow \Delta C(n)=\Phi_{1}\left(n_{0}, n+1\right) F(n) \Phi_{2}^{*}\left(n_{0}, n+1\right) \\
& \Leftrightarrow C(n)=C_{n_{0}}+\sum_{j=n_{0}}^{n-1} \Phi_{1}\left(n_{0}, j+1\right) F(j) \Phi_{2}^{*}\left(n_{0}, j+1\right) .
\end{aligned}
$$

Thus,

$$
\begin{aligned}
T(n)= & \Phi_{1}\left(n, n_{0}\right) C_{n_{0}} \Phi_{2}^{*}\left(n, n_{0}\right) \\
& +\Phi_{1}\left(n, n_{0}\right)\left[\sum_{j=n_{0}}^{n-1} \Phi_{1}\left(n_{0}, j+1\right) F(j) \Phi_{2}^{*}\left(n_{0}, j+1\right)\right] \Phi_{2}^{*}\left(n, n_{0}\right) \\
= & \Phi_{1}\left(n, n_{0}\right) C_{n_{0}} \Phi_{2}^{*}\left(n, n_{0}\right)+\bar{T}(n)
\end{aligned}
$$

where

$$
\bar{T}(n)=\sum_{j=n_{0}}^{n-1} \Phi_{1}(n, j+1) F(j) \Phi_{2}^{*}(n, j+1)
$$

Theorem 2.4. Any solution $T(n)$ of the initial value problem associated with the non-homogeneous matrix difference equation (1.1) with the initial condition $T\left(n_{0}\right)=$ $T_{0}$, where $T_{0}$ is a given constant square matrix of order $s$, is of the form

$$
T(n)=\Phi_{1}\left(n, n_{0}\right) T_{0} \Phi_{2}^{*}\left(n, n_{0}\right)+\sum_{j=n_{0}}^{n-1} \Phi_{1}(n, j+1) F(j) \Phi_{2}^{*}(n, j+1) .
$$

\section{TWO-POINT BOUNDARY VALUE PROBLEM}

Consider the two-point boundary value problem associated with the non-homogeneous matrix differential equation (1.1), satisfying the boundary conditions (1.3). 
The general solution $T(n)$ of (1.1) is given by

$$
T(n)=\Phi_{1}\left(n, n_{0}\right) C_{n_{0}} \Phi_{2}^{*}\left(n, n_{0}\right)+\sum_{j=n_{0}}^{n-1} \Phi_{1}(n, j+1) F(j) \Phi_{2}^{*}(n, j+1) .
$$

The boundary conditions (1.3) will be satisfied if

$$
\begin{gathered}
L_{0} \Phi_{1}\left(n_{0}, n_{0}\right) C_{n_{0}} \Phi_{2}^{*}\left(n_{0}, n_{0}\right)+L_{1} \Phi_{1}\left(n_{1}, n_{0}\right) C_{n_{0}} \Phi_{2}^{*}\left(n_{1}, n_{0}\right) \\
=W-L_{1} \sum_{j=n_{0}}^{n-1} \Phi_{1}\left(n_{1}, j+1\right) F(j) \Phi_{2}^{*}\left(n_{1}, j+1\right) .
\end{gathered}
$$

The above equation (3.1) is equivalent to

$$
A_{0} C_{n_{0}} B_{0}+A_{1} C_{n_{0}} B_{1}=X
$$

where

$$
\begin{array}{ll}
A_{0}=L_{0} \Phi_{1}\left(n_{0}, n_{0}\right), & A_{1}=L_{1} \Phi_{1}\left(n_{1}, n_{0}\right), \\
B_{0}=\Phi_{2}^{*}\left(n_{0}, n_{0}\right), & B_{1}=\Phi_{2}^{*}\left(n_{1}, n_{0}\right)
\end{array}
$$

and

$$
X=W-L_{1} \sum_{j=n_{0}}^{n_{1}-1} \Phi_{1}\left(n_{1}, j+1\right) F(j) \Phi_{2}^{*}\left(n_{1}, j+1\right)
$$

are all known square matrices of order $s$. Note that $B_{0}$ and $B_{1}$ are non-singular matrices and we shall be concerned with the general form of $C_{n_{0}}$ satisfying the conditions (3.2). For the analysis of $C_{n_{0}}$ we use the following notation.

If $P, Q \in C^{s \times s}$ are two square matrices of order $s$, then their Kronecker product (or direct product or tensor product) is denoted by $P \otimes Q$, is defined to be the partitioned matrix

$$
P \otimes Q=\left[\begin{array}{cccc}
P_{11} Q & P_{12} Q & \cdots & P_{1 s} Q \\
P_{21} Q & P_{22} Q & \cdots & P_{2 s} Q \\
\cdots & \cdots & \cdots & \cdots \\
\cdots & \cdots & \cdots & \cdots \\
P_{s 1} Q & P_{s 2} Q & \cdots & P_{s s} Q
\end{array}\right]
$$

and is in $C^{s^{2} \times s^{2}}$.

With this one can easily verify that if $G=\left(A_{0} \otimes B_{0}^{*}\right)+\left(A_{1} \otimes B_{1}^{*}\right)$, then (3.2) is equivalent to a system of equations

$$
G c_{n_{0}}=x
$$

where $G$ is an $s^{2} \times s^{2}$ matrix, and $c_{n_{0}}$ and $x$ are $s^{2}$-column vectors corresponding to the matrices $C_{n_{0}}$ and $X$. In fact by viewing (3.3) as a system of $s^{2}$-scalar equations for the elements of $C_{n_{0}},(3.4)$ is exactly the same set of equations written in a vector system. In order to make pronouncements about existence and uniqueness 
techniques for the solution of (3.4), we need some information about the eigenvalues of $G$. We denote the set of all eigenvalues of the matrix $A$ as $\sigma(A)$, the spectrum of $A$.

Method 1. If $A_{0}$ and $B_{0}$ are non-singular matrices, then (3.2) is equivalent to

$$
C_{n_{0}}-P C_{n_{0}} Q=Y
$$

where $P=-A_{0}^{-1} A_{1}, Q=B_{1} B_{0}^{-1}$ and $Y=A_{0}^{-1} X B_{0}^{-1}$.

Now to solve for $C_{n_{0}}$ from (3.5) we have the following analysis:

$$
\begin{aligned}
C_{n_{0}}-P C_{n_{0}} Q=Y & \Leftrightarrow\left[(I \otimes I)-\left(P \otimes Q^{T}\right)\right] c=Y \\
& \Leftrightarrow I c-\left(P \otimes Q^{T}\right) c=y \\
& \Leftrightarrow I c-G c=y, \quad \text { where } G=P \otimes Q^{T} .
\end{aligned}
$$

Putting

$$
C_{n_{0}}=Y+P C_{n_{0}} Q
$$

in the second term on the L.H.S. of (3.5), we get the following equivalent statements:

$$
\begin{aligned}
& C_{n_{0}}-P\left(Y+P C_{n_{0}} Q\right) Q=Y \Leftrightarrow c-G(y+G c)=y, \\
& C_{n_{0}}-P^{2} C_{n_{0}} Q^{2}=Y+P Y Q \Leftrightarrow c-G^{2} c=y+G y .
\end{aligned}
$$

Similarly

$$
\begin{aligned}
& C_{n_{0}}-P^{3} C_{n_{0}} Q^{3}=Y+P Y Q+P^{2} Y Q^{2} \Leftrightarrow c-G^{3} c=y+G y+G^{2} y \\
& \text { n............................................. } \\
& C_{n_{0}}-P^{m} C_{n_{0}} Q^{m}=Y+P Y Q+\cdots+P^{m-1} Y Q^{m-1} \\
& \Leftrightarrow c-G^{m} c=y+G y+\cdots+G^{m-1} y \text {. }
\end{aligned}
$$

If the spectral radii of $P$ and $Q$ are such that $(\rho(P) \rho(Q))<1$, then $P^{m} C_{n_{0}} Q^{m} \rightarrow 0$ as $m \rightarrow \infty$.

In this case,

$$
\begin{aligned}
C_{n_{0}} & =Y+\sum_{m=1}^{\infty} P^{m} Y Q^{m} \\
& =A_{0}^{-1} X B_{0}^{-1}+\sum_{m=1}^{\infty}\left\{(-1)^{m}\left(A_{0}^{-1} A_{1}\right)^{m}\left(A_{0}^{-1} X B_{0}^{-1}\right)\left(B_{1} B_{0}^{-1}\right)^{m}\right\} .
\end{aligned}
$$


Substituting the above value of $C_{n_{0}}$ in the general solution $T(n)$ of (1.1), we get

$$
\begin{aligned}
& T(n)=\Phi_{1}\left(n, n_{0}\right) A_{0}^{-1} X B_{0}^{-1} \Phi_{2}^{*}\left(n, n_{0}\right) \\
& +\Phi_{1}\left(n, n_{0}\right) \sum_{m=1}^{\infty}\left\{(-1)^{m}\left(A_{0}^{-1} A_{1}\right)^{m}\left(A_{0}^{-1} X B_{0}^{-1}\right)\left(B_{1} B_{0}^{-1}\right)^{m}\right\} \Phi_{2}^{*}\left(n, n_{0}\right) \\
& +\sum_{j=n_{0}}^{n-1} \Phi_{1}(n, j+1) F(j) \Phi_{2}^{*}(n, j+1) \\
& =\Phi_{1}\left(n, n_{0}\right)\left\{A_{0}^{-1}\left[W-L_{1} \sum_{j=n_{0}}^{n-1} \Phi_{1}\left(n_{1}, j+1\right) F(j) \Phi_{2}^{*}\left(n_{1}, j+1\right)\right] B_{0}^{-1}\right. \\
& +\sum_{m=1}^{\infty}(-1)^{m}\left(A_{0}^{-1} A_{1}\right)^{m} A_{0}^{-1} \\
& \times\left[W-L_{1} \sum_{j=n_{0}}^{n-1} \Phi_{1}(n, j+1) F(j) \Phi_{2}^{*}(n, j+1)\right] \\
& \left.\times B_{0}^{-1}\left(B_{1} B_{0}^{-1}\right)^{m}\right\} \Phi_{2}^{*}\left(n, n_{0}\right) \\
& +\sum_{j=n_{0}}^{n-1} \Phi_{1}(n, j+1) F(j) \Phi_{2}^{*}(n, j+1) .
\end{aligned}
$$

Method 2. Suppose $A_{0}$ is invertible. Then the system of equations (3.2) is equivalent to

$$
P C_{n_{0}}+C_{n_{0}} Q=Y
$$

where $P=A_{0}^{-1} A_{1}, Q=B_{0} B_{1}^{-1}$ and $Y=A_{0}^{-1} X B_{1}^{-1}$.

One of the most effective methods of solving the matrix equation (3.6) is the Bartels-Stewart algorithm [7]. Key to this technique is the orthogonal reduction of $P$ and $Q$ to triangular form using the QR-algorithm for eigenvalues. The method of finding the general solution of the system (3.6) is the following.

Let $P, Q \in R^{s \times s}$ be given matrices and define the linear transformation $\psi$ : $R^{s \times s} \rightarrow R^{s \times s}$ by $[6]$

$$
\psi\left(C_{n_{0}}\right)=P C_{n_{0}}+C_{n_{0}} Q=Y .
$$

This linear transformation is non-singular if and only if $P$ and $Q$ have no common eigenvalues, i.e., if $\lambda$ is an eigenvalue of $P$ with corresponding eigenvector $u$ and $\mu$ is an eigenvalue of $Q$ with corresponding eigenvector $v$, then $P u V^{T}=\lambda u v^{T}$ and $u v^{T} Q=\mu u v^{T}$. Hence, $P u v^{T}+u v^{T} Q=(\lambda+\mu) u V^{T}$. This implies that $(\lambda+\mu)$ is an eigenvalue of the system (3.7), which can therefore be solved if and only if $\left(\lambda_{i}+\mu_{j}\right) \neq 0$ for all $i, j=1,2, \ldots, s$. Now reducing $P$ and $Q$ to the diagonal form by using similarity transformations, we get

$$
\begin{aligned}
& U^{-1} P U=\operatorname{diag}\left(\lambda_{1}, \lambda_{2}, \ldots, \lambda_{s}\right)=D_{1}, \\
& V^{-1} Q V=\operatorname{diag}\left(\mu_{1}, \mu_{2}, \ldots, \mu_{s}\right)=D_{2} .
\end{aligned}
$$


Then (3.7) is equivalent to

$$
\left(U^{-1} P U\right)\left(U^{-1} C_{n_{0}} V\right)+\left(U^{-1} C_{n_{0}} V\right)\left(V^{-1} V\right)=U^{-1} Y V .
$$

Solving this system involves four steps.

Step 1. By using similarity transformations, reduce $P$ and $Q$ to diagonal form to get

$$
D_{1}=U^{-1} P U \text { and } D_{2}=V^{-1} Q V .
$$

Step 2. Solve $U \widetilde{F}=Y V$ for $\widetilde{F}$.

Step 3. Solve the transformed system $D_{1} X_{1}+X_{1} D_{2}=F$ for $X_{1}$.

Step 4. Solve the system $C_{n_{0}} V=U X_{1}$ for $C_{n_{0}}$.

From these four steps the solution of the system (3.7) is given by $C_{n_{0}}=U X_{1} V^{-1}$, where $\left(X_{i j}\right)_{1}=\tilde{f}_{i j} /\left(\lambda_{i}+\mu_{j}\right)$, and $\widetilde{F}=\left[\tilde{f}_{i j}\right]=U^{-1} Y V$.

Now substituting the general form of $C_{n_{0}}$ in the general solution $T(n)$ of (1.1), we have

$$
T(n)=\Phi_{1}\left(n, n_{0}\right) U X_{1} V^{-1} \Phi_{2}^{*}\left(n, n_{0}\right)+\sum_{j=n_{0}}^{n-1} \Phi_{1}(n, j+1) F(j) \Phi_{2}^{*}(n, j+1) .
$$

The general solution, in the case of the continuous system

$$
T^{\prime}=A(t) T B(t)+F(t)
$$

in terms of two fundamental matrix solutions of, say, $T^{\prime}=A(t) T$ and $T^{\prime}=B^{*}(t) T$, is not available in the literature. Work in this direction is in progress.

\section{REFERENCES}

1. Ravi P. Agarwal, On multi-point boundary value problems for discrete equations, J. Math. Anal. 96(2) (1983), 520-534. MR 84k:39001

2. V. Lakshmikantham and D. Trigiante, Theory of difference equations: Numerical methods and applications, Academic Press, 1988. MR 89g:39003

3. F. V. Atkinson, Discrete and continuous boundary value problems, Academic Press, New York, 1964. MR 31:416

4. A. Jameson, Solution of the equation $A X+X B=C$ by inversion of an $(m \times m)$ or $(n \times n)$ matrix, SIAM J. Appl. Math. 16(5) 1968, 1010-1023. MR 38:3286

5. P. Lancaster, Explicit solutions of linear matrix equations, SIAM Review 12(4), 1970, 544-566. MR 43:4841

6. G. W. Stewart and R. H. Bartels, A solution of the equation $A X+X B=C$, Common. ACM 15, 1976, 820-826.

7. K. N. Murty, K. R. Prasad and P. V. S. Anand, Two-point boundary value problems associated with Liapunov type matrix difference system, Dynam. Systems Appl. 4 (1995), 205-213. CMP 95:14

Department of Applied Mathematics, Andhra University, Visakhapatnam - 530003 , INDIA

Department of Mathematics, Post Graduate Centre, P. B. Siddhartha College Of Arts \& Science, Vijayawada - 520 010, India 